\title{
Focus on mapping the cells of the body
}

\author{
Consistent integration and annotation of the vast data collected with advanced sequencing technologies will be key \\ to unlocking cell biological insights. We now present a Focus of Perspective articles that discuss ways to improve \\ knowledge consolidation towards generating a human reference atlas.
}

S ingle-cell technologies are an increasingly prominent component of the cell biologist's toolkit. Whether the focus is on the genome, the transcriptome, the methylome or the proteome, single-cell analyses provide snapshots of the cell's building blocks and regulatory networks at unparalleled resolution. Fast-paced progress in sequencing technologies and single-cell approaches have ushered in important developments in computational tools and pipelines to interpret these data and delineate cellular composition, functions, lineage hierarchies and interaction networks. These data-rich disciplines have deepened our knowledge about the composition of the human body. Single-cell analysis is playing a large part in facilitating more accurate models of healthy, diseased or developing organisms through comparisons with cellular reference maps. Combined with genetic lineage-tracking methods, sequencing approaches are helping to identify and characterize rare cell types that were previously outside the range of detection.

A key objective of developmental and cell biologists has been to use single-cell approaches to reconstruct the cellular landscapes of major human organs and provide high-resolution reference maps for the approximately 37.2 trillion cells of the human body. An undertaking of this scope can only be accomplished collaboratively.

So far, a network of 16 consortia is contributing to assemble a human cell atlas and to generate molecular profiles for 30 organs. However, as participating groups and omics technologies increase in number and capabilities, the generated data are reaching unprecedented amounts. There are pressing needs to coordinate and standardize data collection and downstream analysis, and also to formalize the knowledge gained from varied approaches, make it available in public databases, and ensure it is structured, annotated and validated appropriately.
To highlight recent progress in the generation of a human cell atlas from single-cell and spatial omics analyses, we present our Focus, 'Mapping the cells of the body'. The Focus features two specially commissioned Perspective articles published in this issue that discuss progress towards a human reference atlas and a unified ontology that consolidates knowledge about cell types across biological domains. The Focus is hosted on a dedicated page and includes a library of related research articles, research highlights and commentaries published in Nature Cell Biology.

Teichmann and colleagues review progress made in the international collaborative consortium of the Human Cell Atlas (HCA). The HCA was founded in 2016 and includes over 2,000 scientists, making consistent documentation and data reporting a mammoth task. All multi-omics data generated by the consortium are processed by uniform pipelines and presented in a public database. Whether newly discovered or long-established, cell types have to be defined in terms of their anatomical location, lineages, functions and gene expression profiles. Reporting must be done consistently across different studies and laboratories. Naming conventions and cell type classifications have not always been regulated, and newly generated data are not always easily integrated with classical knowledge and established findings. Teichmann and colleagues discuss how a formalized and coordinated cell ontology can provide powerful ways to resolve some of these problems and facilitate the productive interrogation of HCA data.

As a practical example of ontology integration, Börner and colleagues review efforts made by members of 16 international consortia to assemble and connect information about human anatomical structures, cell types, and biomarkers $($ ASCT+B) within a set of ASCT +B tables linked to $3 \mathrm{D}$ representations of anatomy. So far, 11 master tables that cover major organs are available online. Data entries in these tables are mapped using established ontologies such as Cell Ontology or Uberon, with each anatomical structure, cell type or biomarker linked to a resolvable ID. The tables can be used to annotate single-cell sequencing data or explore ongoing progress in the development of atlases. The tables are associated with $3 \mathrm{D}$ anatomical reference maps, which provide visual representations of organs onto which data can be mapped. The Human BioMolecular Atlas Program (HuBMAP) consortium offers a digital visualization tool called ASCT+B Reporter that lets users browse the relationships between entries and two user interfaces for the registration of tissue data and exploration of the tissue dataset in the context of human anatomy.

Beyond the realm of large atlas-generating consortia, single-cell technologies provide opportunities to study and answer cell biological questions in specialized settings. The diverse research articles in our collection are evidence of the broad scope of disciplines that benefit from single-cell technologies. Single-cell analyses have been used to interrogate cellular behaviour across a broad and expanding range of contexts including haematopoiesis, cancer progression, transplantation, organogenesis, embryogenesis and in various model organisms.

At Nature Cell Biology, we are keenly looking forward to the diverse cell types, hierarchies, functions and relationships that still await discovery. We thank our authors and reviewers for their invaluable contributions that enhance the research of this community. We hope that this Focus will provide helpful insights into the current state of this field and its next steps to ensure that data collected in the future are put to optimal use.

Published online: 8 November 2021 https://doi.org/10.1038/s41556-021-00794-8 